\title{
An Improvement of Twisted Ate Pairing with Barreto-Naehrig Curve by using Frobenius Mapping
}

\author{
Yumi Sakemi, Hidehiro Kato, Yasuyuki Nogami and Yoshitaka Morikawa \\ Okayama University \\ Japan
}

\section{Introduction}

Recently, pairing-based cryptographic applications such as ID-based cryptography (Boneh et al., 2001) and group signature scheme (Nakanishi \& Funabiki, 2005) have received much attention. In order to make it practical, various pairings such as Ate pairing (Cohen \& Frey, 2005), subfield-twisted pairing (Matsuda et al., 2007) and subfield-twisted Ate pairing (Devegili et al., 2007) have been proposed. This paper focuses on twisted-Ate pairing with BarretoNaehrig (BN) curve (Barreto \& Naehrig, 2005). As a typical feature of BN curve whose embedding degree is 12, its characteristic $p$, its order $r$, and Frobenius trace $t$ are respectively given with integer variable $\chi$ as follows.

$$
\begin{gathered}
p(\chi)=36 \chi^{4}-36 \chi^{3}+24 \chi^{2}-6 \chi+1, \\
r(\chi)=36 \chi^{4}-36 \chi^{3}+18 \chi^{2}-6 \chi+1, \\
t(\chi)=6 \chi^{2}+1 .
\end{gathered}
$$

Pairing calculation usually consists of two parts, one is Miller's algorithm calculation and the other is so-called final exponentiation. Let $E$ be BN curve of characteristic $p$, Miller's algorithm of twisted-Ate pairing calculates $f_{s, P}(Q) f_{s, P}(Q)$, where $s$ is given by $(t-1)^{2} \bmod r$, $P$ and $Q$ are rational points in certain subgroups of order $r$ in $E\left(F_{p}\right)$ and $E\left(F_{p^{12}}\right)$, respectively. In this case, $(t-1)^{2} \bmod r$ becomes

$$
(t-1)^{2} \bmod r=36 \chi^{3}-18 \chi^{2}+6 \chi-1
$$

it corresponds to the number of iterations of Miller's algorithm. In addition, the hamming weight of $(t-1)^{2} \bmod r$ is preferred to be small for Miller's algorithm to be fast carried out. This paper proposes an improvement of Miller's algorithm.

In the improvement, we use the following relations:

$$
\begin{gathered}
(t-1)^{2} \equiv 36 \chi^{3}-18 \chi^{2}+6 \chi-1 \\
\equiv 6 \chi^{2}(6 \chi-3)+6 \chi-1 \equiv p(6 \chi-3)+6 \chi-1 \bmod r
\end{gathered}
$$


,where $p \equiv t-1=6 \chi^{2} \bmod r$.First, calculate $f_{6 \chi-3, P}(Q)$ by Miller's algorithm, then calculate $f_{6 \chi-1, P}(Q)$ by using the result of the preceding calculation. Then, using the result, calculate $f_{p,(6 \chi-3) P}(Q)$ for which Frobenius mapping in extension field $F_{p^{12}}$ with respect to prime field $F_{p}$ is efficiently applied. In detail, since $p$ is the characteristic of $F_{p^{m}}$, Frobenius mapping does not need any arithmetic operations when the extension field has fast Frobenius mapping such as OEF (Bailey \& Paar, 1998). After that, the authors show some simulation results from which we find that the improvement shown in this paper efficiently accelerates twisted-Ate pairing including final exponentiation about $14.1 \%$.

Throughout this paper, $p$ and $k$ denote characteristic and extension degree, respectively. $F_{p^{k}}$ denotes $k$-th extension field over $F_{p}$ and $F_{p^{k}}{ }^{*}$ denotes its multiplicative group.

\section{Fundamantals}

This section briefly reviews elliptic curve, twisted-Ate pairing, and divisor theorem.

\subsection{Elliptic curve and BN curve}

Let $F_{p}$ be prime field and $E$ be an elliptic curve over $F_{p} . E\left(F_{p}\right)$ that is the set of rational points on the curve, including the infinity point $\mathrm{O}$, forms an additive Abelian group. Let $\# E\left(F_{p}\right)$ be its order, consider a large prime number $r$ that divides $\# E\left(F_{p}\right)$. The smallest positive integer $k$ such that $r$ divides $p^{k-1}$ is especially called embedding degree. One can consider a pairing such as Tate and Ate pairings on $E\left(F_{p^{k}}\right)$.

Usually, \#E(F $\left.F_{p}\right)$ is written as

$$
\# E\left(F_{p}\right)=p+1-t,
$$

where $t$ is the Frobenius trace of $E\left(F_{p}\right)$. Characteristic $p$ and Frobenius trace $t$ of Barreto-Naehrig (BN) curve (Barreto \& Naehrig, 2005) are given by using an integer variable $\chi$ as Eqs.(1). In addition, BN curve $E$ is written as

$$
E\left(F_{p}\right): y^{2}=x^{3}+b, \quad b \in F_{p}
$$

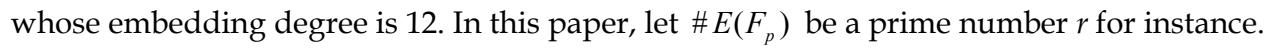

\subsection{Twisted ate pairing with BN curve}

Let $\phi$ be Frobenius endomorphism, i.e.,

$$
\phi: E\left(F_{p^{12}}\right) \rightarrow E\left(F_{p^{12}}\right):(x, y) \rightarrow\left(x^{p}, y^{p}\right),
$$

Then, in the case of BN curve, let $G_{1}$ and $G_{2}$ be

$$
\begin{gathered}
G_{1}=E[r] \cap \operatorname{Ker}(\phi-[1]), \\
G_{2}=E[r] \cap \operatorname{Ker}\left(\left[\xi_{6}\right] \phi^{2}-[1]\right)
\end{gathered}
$$

where $\xi_{6}$ is a primitive 6-th root of unity and let $P \in G_{1}$ and $Q \in G_{2}$, twisted-Ate pairing $\alpha(\cdot, \cdot)$ is defined as 


$$
\alpha(\cdot, \cdot):\left\{\begin{array}{c}
G_{1} \times G_{2} \rightarrow F_{p^{12}}^{*} /\left(F_{p^{12}}^{*}\right)^{r} \\
(P, Q) \mapsto f_{s, P}(Q)^{\left(p^{12}-1\right) / r} .
\end{array}\right.
$$

$A=f_{s, P}(Q)$ is usually calculated by Miller's algorithm (Devegili et al., 2007), then so--called final exponentiation $A^{\left(p^{12}-1\right) / r}$ follows. The number of calculation loops of Miller's algorithm of twisted-Ate pairing with BN curve is determined by $\left\lfloor\log _{2} s\right\rfloor$, where $s$ is, in this case, given by

$$
s=(t-1)^{2} \bmod r=36 \chi^{3}-18 \chi^{2}+6 \chi-1 .
$$

It is said that calculation cost of Miller's Algorithm is about twice of that of final exponentiation.

\subsection{Divisor}

Let $D$ be the principal divisor of $Q \in E$ given as

$$
D=(Q)-(\mathrm{O})=(Q)-(\mathrm{O})+\operatorname{div}(1) .
$$

For scalars $a, b \in Z$, let $a D$ and $b D$ be written as

$$
a D=(a Q)-(\mathrm{O})+\operatorname{div}\left(f_{a, Q}\right), \quad b D=(b Q)-(\mathrm{O})+\operatorname{div}\left(f_{b, Q}\right),
$$

where $f_{a, Q}$ and $f_{b, Q}$ are the rational functions for $a D$ and $b D$, respectively. Then, addition for divisors is given as

$$
a D+b D=(a Q)+(b Q)-(\mathrm{O})+\operatorname{div}\left(f_{a, Q} \cdot f_{b, Q} \cdot g_{a Q, b Q}\right),
$$

where $g_{a Q, b Q}=l_{a Q, b Q} / v_{a Q+b Q}, l_{a Q, b Q}$ denotes the line passing through two points $a Q, b Q$, and $v_{a Q+b Q}$ denotes the vertical line passing through $a Q+b Q$. Moreover, the following relation holds.

$$
a(b D)=\sum_{i=0}^{a-1}(b Q)-a(\mathrm{O})+\operatorname{div}\left(f_{b, Q}^{a} \cdot f_{a, b Q}\right) .
$$

Thus, let $(a+b) D$ and $(a b) D$ be written as

$$
\begin{gathered}
(a+b) D=((a+b) Q)-(\mathrm{O})+\operatorname{div}\left(f_{a+b, Q}\right), \\
(a b) D=(a b Q)-(\mathrm{O})+\operatorname{div}\left(f_{a b, Q}\right) .
\end{gathered}
$$

we have the following relation.

$$
f_{a+b, Q}=f_{a, Q} \cdot f_{b, Q} \cdot g_{a Q, b Q}, \quad f_{a b, Q}=f_{b, Q}{ }^{a} \cdot f_{a, b Q}=f_{a, Q}{ }^{b} \cdot f_{b, a Q} .
$$

Miller's algorithm calculates $f_{s, Q}$ efficiently.

\section{Main proposal}

First, this section briefly goes over Miller's algorithm. Then, an improvement of twisted-Ate pairing with $\mathrm{BN}$ curve of embedding degree 12 is proposed. 


\subsection{Introduction of Miller's algorithm}

Several improvements for Miller's algorithm have been given. Barreto et al. proposed reduced Miller's algorithm. Fig. 1 shows the calculation flow of reduced Miller's algorithm for $f_{s, P}(Q)$. It consists of functions shown in Algorithm 1 and Algorithm 2, see Table 1.

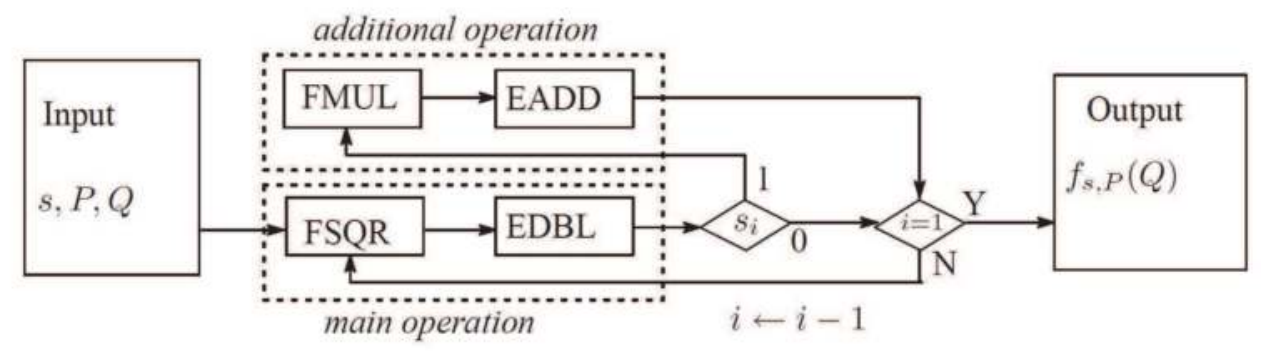

Fig. 1. Calculation flow of Miller's algorithm

In the case of twisted-Ate pairing, let $P \in G_{1}, Q \in G_{2}$ and $s$ be given by Eq.(9), $f_{s, P}(Q)$ becomes an element in $F_{p^{k}}{ }^{*}$. In Fig. 1, $s_{i}$ is the $i$-th bit of the binary representation of $s$ from the lower, FMUL and FSQR denote multiplication and squaring over $F_{p^{12}}$, EADD and EDBL denote elliptic curve addition and doubling over $G_{1}$. As shown in the algorithm, main operation is repeated $\left\lfloor\log _{2} s\right\rfloor$ times but additional operation is only carried out when $s_{i}$ is 1 . Thus, the calculation cost of Miller's Algorithm can be reduced by reducing the number of additional operations.

\begin{aligned} & \hline \hline $\begin{array}{r}\text { Input: } \\ \text { Output: }\end{array} T \in G_{1}, Q \in G_{2} \\ &$\hline \multicolumn{1}{c}{ FSQR } \\ & \hline 1. $\lambda_{T, T} \leftarrow\left(3 x_{T}^{2}\right) /\left(2 y_{T}\right) \\ &$ 2. $l_{T, T}(Q) \leftarrow\left(x_{Q}-x_{T}\right) \lambda_{T, T}-\left(y_{Q}-y_{T}\right) \\ &$ 3. $f \leftarrow f^{2} \cdot l_{T, T}(Q) / v_{T+T}(Q) \\ &$ 4. return $f \\ &$\hline \multicolumn{1}{c}{ EDBL } \\ & \hline 5. $x_{2 T} \leftarrow \lambda_{T, T}^{2}-2 x_{T} \\ &$ 6. $y_{2 T} \leftarrow\left(x_{T}-x_{2 T}\right) \lambda_{T, T}-y_{T} \\ &$ 7. Return $T \leftarrow 2 T \\ &$\hline \hline\end{aligned}

Algorithm 1. FSQR and EDBL of Fig. 1

\subsection{Proposed method}

$f_{A, P}=f_{B, P}$ means $f_{A, P}^{\left(p^{12}-1\right) / r}=f_{B, P}{ }^{\left(p^{12}-1\right) / r}$ in what follows, where $f_{A, P}$ and $f_{B, P}$ are the rational functions of divisors, respectively. Miller's algorithm of twisted-Ate pairing with BN curve calculates $f_{s, P}(Q)$, where $s$ is given as

$$
s=(t-1)^{2}=36 \chi^{3}-18 \chi^{2}+6 \chi-1 \bmod r .
$$




\begin{aligned} & \hline \hline $\begin{array}{r}\text { Input: } \\ \text { Output: }\end{array} P \in G_{1}, Q \in G_{2} \\ &$\hline \multicolumn{1}{c}{ FMUL } \\ & \hline 1. $\lambda_{T, P} \leftarrow\left(y_{P}-y_{T}\right) /\left(x_{P}-x_{T}\right) \\ &$ 2. $l_{T, P}(Q) \leftarrow\left(x_{Q}-x_{P}\right) \lambda_{T, P}-\left(y_{Q}-y_{P}\right) \\ &$ 3. $f \leftarrow f \cdot l_{T, P}(Q) / v_{T+P}(Q) \\ &$ 4. return $f \\ &$\hline EADD \\ & \hline 5. $x_{T+P} \leftarrow \lambda_{T, P}^{2}-x_{T}-x_{P} \\ &$ 6. $y_{T+P} \leftarrow\left(x_{P}-x_{T+P}\right) \lambda_{T, P}-y_{P} \\ &$ 7. return $T \leftarrow 2 T \\ &$\hline \hline\end{aligned}

Algorithm 2. FMUL and EADD of Fig. 1

\begin{aligned} \hline$s_{i}: &$ the $i$-th bit of the binary representation of $s$ from the lower. \\ $l_{T, T}: &$ the tangent line at $T \\ . l_{T, P}: &$ the line passing through $T$ and $P \\ . v_{T+T}: &$ the vertical line passing through $2 T \\ . v_{T+P} &$ the vertical line passing through $T+P \\ . \lambda_{T, T}: &$ the slope of the tangent line $l_{T, T} \\ . \lambda_{T, P}: &$ the slope of the line $l_{T, P}\end{aligned}$.

Table 1. Notations used in Algorithms 1, 2, and 3

\begin{tabular}{|c|c|c|}
\hline$r(\chi)$ & $\chi$ & $\mathrm{Hw}\left(s^{*}\right)$ \\
\hline \hline & $2^{62+2^{46+229}}$ & 83 \\
254 & $2^{64+235+2^{24}}$ & 82 \\
Bits & $2^{62+255+1}$ & 36 \\
& $-2^{62-2} 2^{41-223}$ & 43 \\
\hline
\end{tabular}

${ }^{*} s=(t-1)^{2} \bmod r=36 \chi^{3}-18 \chi^{2+6 \chi-1}$

Table 2. $\chi$ of small Hamming weight that gives BN curve of 254 bits prime order

The proposed method calculates $f_{s, P}(Q)$ using the following relations:

$$
\begin{gathered}
p \equiv t-1=6 \chi^{2} \bmod r \\
s \equiv 6 \chi^{2}(6 \chi-3)+6 \chi-1 \equiv p(6 \chi-3)+6 \chi-1 \bmod r .
\end{gathered}
$$

Using $\chi$ of small Hamming weight, first calculate $f_{6 \chi-3, P}(Q)$ and then calculate $f_{6 \chi-1, P}(Q)$ by using the result of the preceding calculation. Then, by calculating $f_{p,(6 x-3) P}(Q)$ for which Frobenius mapping is efficiently applied, the number of additional operations is substantially reduced. In detail, let $\chi^{\prime}=2 \chi-1$, calculate $f_{\chi^{\prime}, P}(Q)$ by Miller's algorithm. Then, calculate $f_{6 \chi-3, P}(Q)$ as

$$
f_{6 \chi-3, P}=f_{\chi^{\prime}, P}^{3} \cdot g_{\chi^{\prime} P, \chi^{\prime} P} \cdot g_{2 \chi^{\prime} P, \chi^{\prime} P}
$$


Since $6 \chi-1=(6 \chi-3)+2, f_{6 \chi-1, P}(Q)$ is given as

$$
f_{6 \chi-1, P}=f_{6 \chi-3, P} \cdot f_{2, P} \cdot g_{(6 \chi-3) P, 2 P} .
$$

Then, calculate $f_{\left(6 \chi^{3}\right) \cdot 6 \chi^{2}, P}$ by using $f_{6 \chi-3, P}$. Algorithm 3 shows Miller's algorithm whose initial value of $f$ is $f$. Though it can be calculated by Algorithm 3 as

$$
f_{(6 \chi-3) \cdot 6 \chi^{2}, P}=\left.f_{6 \chi^{2},(6 \chi-3) P}\right|_{f^{\prime}=f_{(6 \chi-3), P}},
$$

according to Eq.(16a), this paper calculates it by Algorithm 3 as follows.

$$
f_{(6 \chi-3) \cdot 6 \chi^{2}, P}=\left.f_{(6 \chi-3), P}^{6 \chi^{2}} \cdot f_{6 \chi^{2},(6 \chi-3) P}\right|_{f^{\prime}=1}=\left.f_{(6 \chi-3), P}^{p} \cdot f_{6 \chi^{2},(6 \chi-3) P}\right|_{f^{\prime}=1}
$$

Finally, we have

$$
f_{6 \chi^{2}(6 \chi-3)+6 \chi-1, P}=f_{(6 \chi-1), P} \cdot f_{(6 \chi-3), P}^{p} \cdot\left(\left.f_{6 \chi^{2},(6 \chi-3) P}\right|_{f^{\prime}=1}\right) \cdot g_{(6 \chi-1) P,(6 \chi-3) P} .
$$

The proposed method has the following advantages.

- $\quad \chi$ of small hamming weight efficiently works.

- It can reduce a multiplication in $F_{p^{12}}$ at Step6 of Algorithm 3 by Frobenius mapping.

\begin{tabular}{rc}
\hline \hline Input: & $P \in G_{1}, Q \in G_{2}, f^{\prime} \in F_{p^{12}}$ \\
Output: & $f_{\chi, P^{\prime}}(Q)$ \\
\hline 1. & $f \leftarrow f^{\prime}, T \leftarrow P$ \\
2. & For $i=\left\lfloor\log _{2}(s)\right\rfloor$ downto $1:$ \\
3. & $f \leftarrow f^{2} \cdot l_{T, T}(Q) / v_{T+T}(Q)$ \\
4. & $T \leftarrow 2 T$ \\
5. & If $s_{i}=1$, then : \\
6. & $f \leftarrow f \cdot f^{\prime} \cdot l_{T, P}(Q) / v_{T+P}(Q)$ \\
7. & $T \leftarrow T+P$ \\
8. & return $f$ \\
\hline \hline
\end{tabular}

Algorithm 3. Miller's Algorithm whose initial value of $f$ is $f^{\prime}$.

\section{Experimental result}

In order to show the efficiency of the proposed method, the authors simulated twisted-Ate pairing with BN curve of order $r \approx 2^{254}$. In this simulation, the authors used $\chi$ and BN curve shown in Table 3. Table 4 shows the simulation result.

As a reference, Table 5 shows timings of multiplication (mul), inversion (inv) in each subfield of $F_{p^{12}}$ and squaring (sqr) in $F_{p^{12}}$. According to Table 4, in the cases of $r \approx 2^{254}$, the proposed method reduced the calculation times of Miller's algorithm by $18.0 \%$. 


\begin{tabular}{|c||c|}
\hline size of $p, r$ & 254 bits \\
\hline BN curve & $y^{2}=x^{3}+10$ \\
$\chi$ & $2^{64+22^{35}+2^{24}}$ \\
$\operatorname{Hw}(s)$ & 82 \\
$\operatorname{Hw}(\chi)$ & 3 \\
\hline
\end{tabular}

Table 3. Parameters of twisted-Ate pairing

\begin{tabular}{|l|l||c|}
\hline \multicolumn{2}{|c||}{$p, r$} & 254 bits \\
\hline \multirow{2}{*}{ Miller's part } & Conventional & 14.5 \\
\cline { 2 - 3 } & Proposed & 11.8 \\
\hline final exponentiation & 4.45 \\
\hline \multirow{2}{*}{ Total } & Conventional & 19.0 \\
\cline { 2 - 3 } & Proposed & 16.3 \\
\hline \multirow{2}{*}{$\begin{array}{l}\text { Elliptic curve } \\
\text { scalar multiplication* }\end{array}$} & $G_{1} \in E\left(F_{p}\right)^{* *}$ & 2.31 \\
\cline { 2 - 3 } & $G_{2} \in E^{\prime}\left(F_{p^{2}}\right)$ & 7.01 \\
\hline
\end{tabular}

* Average timings with random scalars and exponents of

** Projective coordinates are used.

Remark : Pentium4 (3.6GHz), C language, and GMP 4.2.2 library are used.

Table 4. Comparison of timings [ms]

\begin{tabular}{|l|c||c|}
\hline$F_{p}$ & mul & 0.65 \\
& inv & 8.43 \\
\hline$F_{p^{2}}$ & mul & 1.65 \\
& inv & 11.4 \\
\hline$F_{p^{4}}$ & mul & 4.39 \\
& inv & 19.6 \\
\hline$F_{p^{6}}$ & mul & 7.78 \\
& inv & 32.4 \\
\hline \multirow{3}{*}{$F_{p^{12}}$} & mul & 21.6 \\
& inv & 80.3 \\
\hline
\end{tabular}

Remark: Pentium4 (3.6GHz), C

language, and GMP 4.2.2 library are used.

Table 5. Timings of operations in subfield ( $p$ : 254 bit prime number $)[\mu \mathrm{s}]$

\section{Conclusion}

This paper has proposed an improvement of twisted-Ate pairing with Barreto-Naehrig curve so as to efficiently use Frobenius mapping with respect to prime field. Then, this paper showed some simulation result by which it was shown that the improvement accelerated twisted-Ate pairing including final exponentiation about $14.1 \%$. 


\section{Acknowledgement}

This work is supported by "'Strategic Information and Communications R\&D Promotion Programme" from the Ministry of Internal Affairs and Communications, Japan.

\section{References}

Bailey, D. \& Paar, C. (1998). Optimal Extension Fields for Fast Arithmetic in Public-Key Algorithms, Proceedings of CRYPT'98, pp.472-485, ISBN: 978-3-540-64892-5, USA, August 1998, Springer-Verlag, Santa Barbara, California

Barreto, P. S. L. M. \& Naehrig, M. (2006). Pairing-Friendly Elliptic Curves of Prime Order, Proceedings of SAC2005, pp. 319-331, ISBN: 978-3-540-33108-7, Canada, August 2005, Springer-Verlag, Kingston

Boneh, D.; Lynn, B. \& Shacham, H. (2001). Short signatures from the Weil pairing, Proceedings of Asiacrypt2001, pp. 514-532, ISBN: 978-3-540-42987-6, Australia, December 2001, Springer-Verlag, Gold Coast

Cohen, H. \& Frey, G. (2005). Handbook of Elliptic and Hyperelliptic Curve Cryptography, Chapman \& Hall CRC, ISBN: 1584885181

Devegili, A. J.; Scott, M. \& Dahab, R. (2007). Implementing Cryptographic Pairings over Barreto-Naehrig Curves, Proceedings of Pairing2007, pp. 197-207, ISBN: 978-3-54073488-8, Japan, July 2007, Springer-Verlag, Tokyo

GNU Multiple Precision Arithmetic Library, http://gmplib.org/

Hess, F.; Smart, N.; \& Vercauteren, F. (2006). The Eta Pairing Revisited, IEEE Trans. Information Theory, Vol. 52, No. 10, October 2006, pp. 4595-4602, ISSN: 0018-9448

Matsuda, S.; Kanayama, N; Hess, F.; \& Okamoto, E. (2007). Optimised Versions of the Ate and Twisted Ate Pairings, Proceedings of $11^{\text {th }}$ IMA International Conference, pp. 302312, ISBN: 978-3-540-77272-9, UK, December 2007, Springer-Verlag, Cirencester.

Nakanishi, T. \& Funabiki, N. (2005). Verifier-Local Revocation Group Signature Schemes with Backward Unlinkability from Bilinear Maps, Proceedings of Asiacrypt2005, pp. 443-454, ISBN: 978-3-540-30684-9, India, December 2005, Springer-Verlag, Chennai 


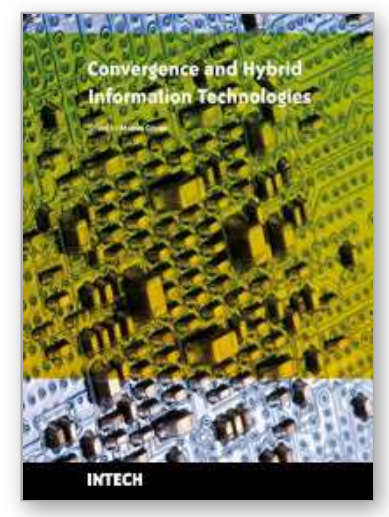

\author{
Convergence and Hybrid Information Technologies \\ Edited by Marius Crisan
}

ISBN 978-953-307-068-1

Hard cover, 426 pages

Publisher InTech

Published online 01, March, 2010

Published in print edition March, 2010

Starting a journey on the new path of converging information technologies is the aim of the present book. Extended on 27 chapters, the book provides the reader with some leading-edge research results regarding algorithms and information models, software frameworks, multimedia, information security, communication networks, and applications. Information technologies are only at the dawn of a massive transformation and adaptation to the complex demands of the new upcoming information society. It is not possible to achieve a thorough view of the field in one book. Nonetheless, the editor hopes that the book can at least offer the first step into the convergence domain of information technologies, and the reader will find it instructive and stimulating.

\title{
How to reference
}

In order to correctly reference this scholarly work, feel free to copy and paste the following:

Yumi Sakemi, Hidehiro Kato, Yasuyuki Nogami and Yoshitaka Morikawa (2010). An Improvement of Twisted Ate Pairing with Barreto-Naehrig Curve by using Frobenius Mapping, Convergence and Hybrid Information Technologies, Marius Crisan (Ed.), ISBN: 978-953-307-068-1, InTech, Available from:

http://www.intechopen.com/books/convergence-and-hybrid-information-technologies/an-improvement-oftwisted-ate-pairing-with-barreto-naehrig-curve-by-using-frobenius-mapping

\section{INTECH}

open science | open minds

\author{
InTech Europe \\ University Campus STeP Ri \\ Slavka Krautzeka 83/A \\ 51000 Rijeka, Croatia \\ Phone: +385 (51) 770447 \\ Fax: +385 (51) 686166 \\ www.intechopen.com
}

\author{
InTech China \\ Unit 405, Office Block, Hotel Equatorial Shanghai \\ No.65, Yan An Road (West), Shanghai, 200040, China \\ 中国上海市延安西路65号上海国际贵都大饭店办公楼405单元 \\ Phone: +86-21-62489820 \\ Fax: +86-21-62489821
}


(C) 2010 The Author(s). Licensee IntechOpen. This chapter is distributed under the terms of the Creative Commons Attribution-NonCommercialShareAlike-3.0 License, which permits use, distribution and reproduction for non-commercial purposes, provided the original is properly cited and derivative works building on this content are distributed under the same license. 